\title{
Epileptogenesis, Ictogenesis and the Design of Future Antiepileptic Drugs
}

\author{
Donald F. Weaver
}

\begin{abstract}
There is still no medical cure for epilepsy. Clinical epileptology is in need of a "paradigm shift" when it comes to the continuing development of therapeutics. An important first step in this conceptual evolution is differentiating between the notions of ictogenesis and epileptogenesis. All traditional therapeutics are anti-ictogenic, not antiepileptogenic. The future of antiepileptic drug development lies in the discovery of antiepileptogenics. Just as aspirin is not the drug of choice for meningitis, an anticonvulsant is not the drug of choice for epilepsy. Drug design for epilepsy needs to discover a penicillin, not more aspirins.
\end{abstract}

RÉSUMÉ: Épileptogenèse, ictogenèse et conception de médicaments antiépileptiques. Il n'existe pas encore de traitement médical curatif de l'épilepsie. L'épileptologie clinique aurait besoin d'un changement de perspective quant au développement de nouveaux traitements. Un premier pas important dans cette évolution conceptuelle est la distinction entre les notions d'ictogenèse et d'épileptogenèse. Tous les traitements traditionnels sont anti-ictogènes et non antiépileptogènes. L'avenir du développement des médicaments antiépileptiques réside dans la découverte d'antiépileptogènes. Tout comme l'aspirine n'est pas le médicament de choix pour la méningite, un anticonvulsivant n'est pas le médicament de choix pour traiter l'épilepsie. Ce dont nous avons besoin dans la conception de médicaments pour traiter l'épilepsie, ce n'est pas la découverte de plus d'aspirine mais la découverte d'une pénicilline.

Can. J. Neurol. Sci. 2003; 30: 4-7

Despite the continuing introduction of so-called antiepileptic drugs (AEDs), there is still no medical cure for epilepsy, no receptor-specific drug that can either prevent epilepsy or alter its natural course. This is significant since epilepsy not only is the most common serious neurologic disorder affecting patients of all ages but also is a prototypic clinical disorder for probing fundamental neurobiological processes, such as the mechanisms of core human consciousness. The currently available anticonvulsant drugs, whether old or new, are little more than "symptomatic" agents that suppress the symptoms of epilepsy (i.e. seizures) while failing to contend with the underlying pathological process that initially caused (or continues to cause) the predisposition to seizures. Even as symptomatic agents, the existing therapies are far from ideal, being consistently effective in fewer than $65 \%$ of patients and tending to produce a variety of side-effects in more than $50 \%$ of patients. Although the need to discover pioneering "curative" therapeutics for epilepsy is clinically obvious, the direction to be pursued in the realization of such agents is not apparent. With an existing drug selection populated by a diversity of traditional agents (e.g. phenytoin, carbamazepine), new agents (e.g. lamotrigine, topiramate) and emerging agents (e.g. zonisamide), the interests of the patient population will not be best served by simply more of the same (Figure 1). Abused though the expression may be, it is arguable that the world of clinical epileptology is in need of a "paradigm shift" when it comes to the continuing development of therapeutics.

The failings of traditional anticonvulsant drugs to influence the natural history of epilepsy have been demonstrated repeatedly. As shown by various well-controlled studies, standard agents such as phenytoin have no influence on the delayed development of epilepsy when used prophylactically after a brain insult and do not prevent the progressive pathology that underlies a developing seizure disorder. ${ }^{1,2}$ Indeed, limited evidence suggests that these drugs may be achieving quite the contrary. ${ }^{3}$ Phenytoin prophylaxis, for instance, has been associated with neurobehavioural deterioration in a variety of neuropsychological measures in posttraumatic epilepsy patients; additionally, the discontinuation of either carbamazepine or phenytoin in brain-injured individuals has produced improvement in measures of fluency, memory, and motor performance. Clearly, the search for new agents for epilepsy should probably not use existing anticonvulsant drugs as a

From the Departments of Medicine (Neurology) and Chemistry, Dalhousie University, Halifax, Nova Scotia, Canada.

RECEIVED JANUARY 16, 2002. ACCEPTED IN FINAL FORM JULY 9, 2002.

Reprint requests to: D.F. Weaver, Depts. of Medicine (Neurology) and Chemistry, Chemistry Building, Dalhousie University, Halifax, Nova Scotia, Canada B3H 4J3 
<smiles>O=C1NC(=O)C(c2ccccc2)(c2ccccc2)N1</smiles>

Phenytoin<smiles>NC(=O)N1c2ccccc2C=Cc2ccccc21</smiles>

Carbamazepine<smiles>CCC1(C)CC(=O)NC1=O</smiles>

Ethosuximide<smiles>CCCC(CCC)C(=O)O</smiles>

Valproic acid<smiles>Nc1nnc(-c2cccc(Cl)c2Cl)c(N)n1</smiles>

Lamotrigine<smiles>CC1(C)OC2COC3(COS(N)(=O)=O)OC(C)(C)OC3C2O1</smiles>

Topiramate

Figure 1: Molecular structures of anticonvulsant drugs

starting point in the design process. Central to the discovery of definitive therapeutics, which positively influence the natural history of epilepsy in a curative sense, will be the evolution of concepts concerning the molecular pathogenesis of epilepsy.

A first step in this conceptual evolution of identifying new targets for drug design relevant to partial seizures is differentiating between the notions of "ictogenesis" and "epileptogenesis". A seizure is a single discrete clinical event caused by an excessive electrical discharge from a collection of neurons. Seizures are merely the symptom of epilepsy. Epilepsy, on the other hand, is a dynamic and frequently progressive process characterized by an underlying sequence of pathological transformations whereby normal brain is altered, becoming susceptible to spontaneous, recurrent seizures. Ictogenesis (the initiation and propagation of a seizure in time and space) is a rapid electrical/chemical event occurring over seconds or minutes. Epileptogenesis (the gradual process whereby normal brain is transformed into a state susceptible to spontaneous, episodic, time-limited recurrent seizures through the initiation and maturation of an "epileptogenic focus") is a slow biochemical/histological process occurring insidiously over months to years. Ictogenesis and epileptogenesis have unique differences; not surprisingly, therapeutics targeting these two processes may have definite differences. ${ }^{5}$ (Figure 2.)

Ictogenesis is a fast, short-term electrical/chemical event divided into the rapidly sequential phases of initiation and elaboration; elaboration arises from the extension of the seizure in both time and space. At the molecular level, ictogenesis involves excessive brain electrical discharges propagated by a cascade of chemical events initiated by the sequential opening of voltage gated $\mathrm{Na}^{+}$channels with subsequent involvement of $\mathrm{K}^{+}$ channels and the $\mathrm{Ca}^{2+}$ channel mediated release of neurotransmitters. Logically, diverse mechanisms of action exist for anti-ictogenic (anticonvulsant, antiseizure) drugs. However, the central role of the transmembrane voltage gated $\mathrm{Na}^{+}$channel in ictogenesis has resulted in the majority of the current anticonvulsant drugs (e.g. phenytoin, carbamazepine, valproate, lamotrigine) being targeted against this receptor site.

Epileptogenesis, unlike ictogenesis, is a gradual two phase biochemical/histological process showing dynamic changes over the course of time: Phase 1 - the initiation of the epileptogenic

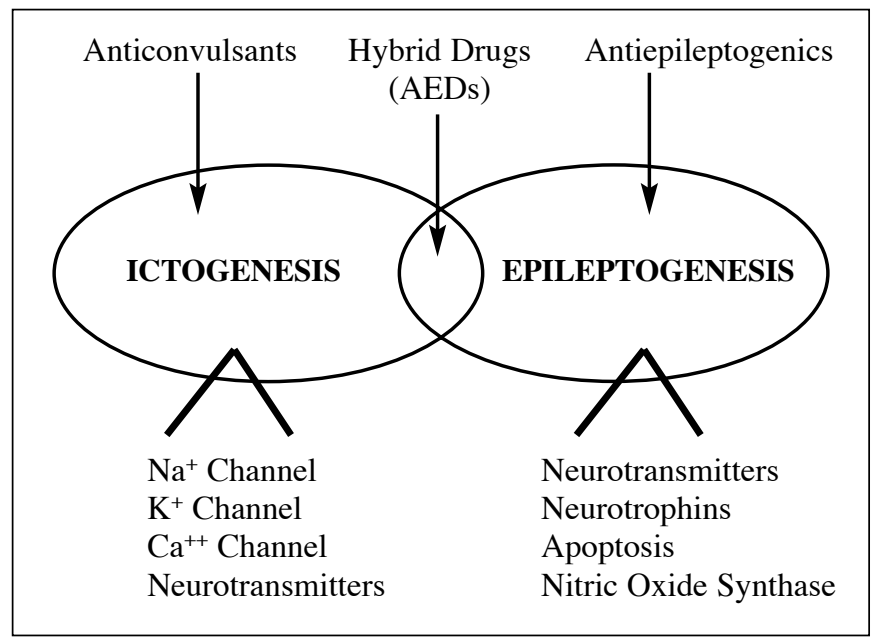

Figure 2: The molecular basis of ictogenesis and epileptogenesis 
focus, and Phase 2 - the maturation of an active epileptogenic focus. ${ }^{5}$ Phase 1 epileptogenesis refers to the events that take place prior to the occurrence of the first seizure. There may be a considerable delay of months to years between the occurrence of the brain injury (e.g. meningitis, trauma) and the onset of recurrent seizures. During this latent period, epileptogenesis is evolving, culminating in active epilepsy in which recurrent seizures occur. Phase 2 epileptogenesis refers to the events that take place after the first seizure(s) has occurred. This also is a long, protracted process in which seizures may become more frequent, more severe, more refractory to treatment, or phenomenologically different in their clinical manifestations.

The cascade of histological/biochemical events which characterize epileptogenesis differ from those of ictogenesis. This is best exemplified by an examination of the pathology of complex partial seizures. At the histological level, epileptogenesis involves cellular alterations (e.g. mesial temporal sclerosis) in a variety of limbic brain structures. The macroscopic features of these alterations include fibrous gliosis with cellular shrinkage and atrophy. Microscopically, there is neuronal loss affecting the pyramidal cell layers in the hippocampal subfields CA1, CA3, and in the dentate gyrus, but sparing the granule cells of the dentate gyrus. There is also significant reorganization of dendritic and axonal projections from pyramidal and other hippocampal neurons, revealing the formation of new synaptic contacts and neuronal processes. At the biochemical level, various theories of epileptogenesis have been put forth, including the mossy fibre sprouting hypothesis and the dormant basket cell hypothesis. The mossy fibre sprouting hypothesis, from Sutula and others, 6,7 postulates an upregulation of excitatory coupling between neurons mediated by $\mathrm{N}$-methyl-D-aspartate glutamatergic receptors which are activated in chronic epileptic brain under circumstances that would not lead to activation in normal brain. In contrast, the dormant basket cell hypothesis, as espoused by Sloviter ${ }^{8}$ and Beckenstein ${ }^{9}$ suggests a downregulation of inhibitory coupling between neurons such that the connections which normally drive $\gamma$-aminobutyric acid [GABA] releasing inhibitory interneurons are disturbed thereby rendering them functionally dormant. In combining these two hypotheses, Jefferys ${ }^{10}$ and Larner ${ }^{11}$ have suggested that susceptibility to abnormal, synchronous firing of neurons (crucial to epileptogenesis) arises from a concurrent enhancement of glutamate mediated excitatory synaptic transmission time-locked to a decrease in GABA-mediated inhibitory synaptic transmission - the resulting imbalance leading to sustained neuronal hyperexcitability, and hence to epileptogenesis.

Although such glutamatergic and GABAergic processes are obvious participants in the molecular mechanism of epileptogenesis, there are other molecular claimants to the throne. For example, in the kindling animal model of epileptogenesis (in which repetitive, subconvulsive, electrical stimulation evokes progressively prolonged electrographic/ behavioural responses that culminate in generalized seizures) neurotrophic peptides, such as nerve growth factor, play a facilitative role in hippocampal synaptic reorganization. Neurotrophins therefore seem to contribute to the evolution of the epileptogenic state. ${ }^{12-15}$ Other molecular processes potentially involved in the excitotoxic cascade of epileptogenesis include cellular $\mathrm{Ca}^{2+}$ influx, apoptosis, nitric oxide synthase overproduction and free radical accumulation. Clearly, the future development of antiepileptogenic agents must exploit the full range of targets, extending from amino acid neurotransmitters to peptidic neuromodulators. Also, since "the epilepsies" are a heterogeneous group of disorders (i.e. inherited vs. acquired; primary generalized vs. partial) it may be necessary to design antiepileptogenic agents which are specific for a particular type of epilepsy.

As an additional complication, there seemingly exists an interdependency between ictogenesis and epileptogenesis (despite their biological uniquenesses and chemical differences). As Gowers ${ }^{16}$ opined in the 1880 s, "seizures beget seizures", thus recognizing that epilepsy is sometimes a progressive disorder. Although somewhat controversial, current experimental evidence (e.g. the kindling model) continues to support the notion that the recurrence of seizures per se can be responsible (at least in part) for progression of epilepsy. Unfortunately, data from human clinical studies are less robust. Clearly, some human epilepsies (e.g. temporal lobe epilepsy) are progressive; clearly, other types are not. Nonetheless, the events of ictogenesis may constitute a sub-set of additive factors contributing to the process of epileptogenesis.

At the present time, there are no clinically proven antiepileptogenic drugs, an observation supported by both experimental and clinical studies. ${ }^{17}$ Currently available so-called "antiepileptic drugs" are not really AEDs; rather, they are simply anti-ictogenic agents with no clinically demonstrated antiepileptogenic efficacy. (Arguably, the term AED should be abandoned as a misnomer. The currently marketed drugs should be referred to by a more accurate designation, such as "antiseizure (anti-ictogenic, anticonvulsant) drugs"; the expression antiepileptogenic drug should be used for agents that suppress epileptogenesis, but not necessarily ictogenesis. The term AED should ideally be reserved for future agents which will be effective against both epilepsy as a process and seizures as events within that process.)

Future drugs for epilepsy must extend beyond the scope of simple ictogenesis and should encompass the greater mandate of epileptogenesis. Since the two processes are separate yet interdependent, future agents should probably not be purely antiepileptogenic, but capable of hybrid antiepileptogenic/antiictogenic activity. The discovery of such hybrid agents demands changes in our conceptual approaches to AED design and discovery, and will require the acceptance of new animal models. Existing animal models, such as the maximal electroshock assay and the pentylenetetrazol assay are models of ictogenesis, not epileptogenesis. The kindling model, though much studied, is controversial and may not be a good model of epileptogenesis. Although more cumbersome, other models such as the pilocarpine-induced spontaneous recurrent seizure model may have to be implemented in the search for new agents which are truly AEDs. ${ }^{18}$ This "paradigm shift" must cross the spectrum from animal studies to human studies, and must be accompanied by subsequent improvements in clinical trial design. Designing human clinical trials to assess novel agents for antiepileptogenic activities will be challenging, potentially involving prolonged observational periods in patient populations susceptible to processes such as delayed posttraumatic epilepsy. Future human 
trials must also incorporate increasingly sophisticated and allencompassing outcome measures which exceed mere seizure counts and which consider the clinical phenomenology of epileptogenesis. Memory disorders, behavioural problems and related neuropsychological issues are equally important byproducts of epileptogenesis; epileptogenesis is a complex neuropathological process and is not simply a generator of seizures. These advances in the design of therapeutics must be accompanied by advances in the design of diagnostics. At the present time, there are no diagnostic tests to ascertain a unique propensity to epileptogenesis in any individual patient experiencing a given CNS insult.

When considered rigorously, all epilepsies are currently "drug resistant" or "intractable to medical management"; the current anticonvulsants, either old or new, are noncurative palliative therapeutics. The need for curative drugs is an emerging neuropharmacologic priority in clinical epileptology. Drug discovery and the identification of targets for rational drug design must take this into consideration. Bacterial meningitis is treated with penicillin in preference to aspirin; so too must epilepsy be treated with more than simply symptomatic therapies. Just as aspirin is not the drug of choice for Staphylococcal meningitis, an anticonvulsant is not the drug of choice for epilepsy. Drug design for epilepsy needs to discover a penicillin, not more aspirins.

\section{ACKNOWLEDGEMENTS:}

DFW acknowledges the financial support of a Tier 1 CRC in Neuroscience at Dalhousie University. DFW's research is supported by operating grants from CIHR and NSERC. This paper is dedicated to Dr. D. Howse, Queen's University, on the occasion of his 65th birthday, who initially motivated the author's interest in anticonvulsant drug design.

\section{REFERENCES}

1. Temkin NR, Dikmen SS, Wilensky AJ, et al. A randomised doubleblind study of phenytoin for the prevention of posttraumatic seizures. N Engl J Med 1990; 323: 497-502.

2. Foy PM, Chadwick DW, Rajgopalan N, Johnson AL, Shaw MD. Do prophylactic anticonvulsant drugs alter the pattern of seizures after craniotomy? J Neurol Neurosurg Psychiatry 1992; 55: 753757.

3. Hernandez TD. Preventing posttraumatic epilepsy after brain injury: weighing the costs and benefits of anticonvulsant prophylaxis. Trends Pharmacol Sci 1997; 18: 59-62.

4. Lothman EW. Basic mechanisms of seizure expression. Epilepsy Res 1996; S11: 9-16.

5. Weaver DF. Applications of computer assisted quantum pharmacology calculations in devising anticonvulsant rational polypharmacy strategies. Epilepsy Res 1996; S11: 95-113.

6. Sutula T, Xiao-Xian H, Cavazos J, et al. Synaptic reorganization in the hippocampus induced by abnormal functional activity. Science 1988; 239: 1147-1150.

7. Sutula T, Cascino G, Cavazos J, et al. Mossy fiber synaptic reorganization in the epileptic human temporal lobe. Ann Neurol 1989; 26: 321-330.

8. Sloviter R. Permanently altered hippocampal structure, excitability, and inhibition after experimental status epilepticus in the rat: the dormant basket cell hypothesis and its possible relevance to temporal lobe epilepsy. Hippocampus 1991; 1: 41-66.

9. Beckenstein J, Lothman EW. Dormancy of inhibitory interneurons in a model of temporal lobe epilepsy. Science 1993; 259: 97-100.

10. Jeffreys JGR. Experimental neurobiology of epilepsies. Curr Opin Neurol Neurosurg 1994; 7: 113-122.

11. Larner AJ. Axonal sprouting and synaptogenesis in temporal lobe epilepsy: possible pathogenetic and therapeutic roles of neurite growth inhibitory factors. Seizure 1995; 4: 249-258.

12. Gall C, Isackson P. Limbic seizures increase neuronal production of messenger RNA for nerve growth factor in adult rat forebrain. Science 1989; 245: 758-761.

13. Bengzon J, Kokaia A, Ernfors P, et al. Regulation of neurotrophin and trkA, trkB and trkC tyrosine kinase receptor messenger RNA in kindling. Neuroscience 1993; 53: 433-446.

14. Lowenstein DH, Seren MS, Longo FM. Prolonged increases in neurotrophic activity associated with kainate-induced hippocampal synaptic reorganization. Neuroscience 1993; 56: 597-604

15. Crutcher KA, Scott SA, Liang S, Evereson WV, Weingartner J. Detection of NGF-like activity in human brain tissue. J Neurosci 1993; 13: 2540-2550.

16. Gower WR. Epilepsy and Other Chronic Convulsive Disorders. London: Churchill, 1881

17. Shinnar S, Berg AT. Does antiepileptic drug therapy prevent the development of chronic epilepsy? Epilepsia 1996; 37: 701-708.

18. Mello LEAM, Cavalheiro EA, Tan AM, et al. Circuit mechanisms of seizures in the pilocarpine model of chronic epilepsy: cell loss and mossy fibre sprouting. Epilepsia 1993; 34: 985-995. 